\title{
Thermal and cutting process simulation analysis of laser assisted milling of Inconel 718
}

\author{
XueFeng $\mathrm{Wu}^{1, \mathrm{a}^{*}}$,Bowen Zhao ${ }^{1, \mathrm{~b}}$ and GaoCheng Feng ${ }^{1, \mathrm{c}}$ \\ ${ }^{1}$ Harbin University of Science and Technology, Harbin, Heilongjiang, China \\ awuxuefeng@hrbust.edu.cn, bhrbust_zhao@126.com, '18772728807@163.com
}

Keywords: Laser assisted milling; thermal simulation; Inconel 718; cutting process simulation

\begin{abstract}
Laser assisted milling (LAML) is a potential method for machining difficult-to-machine materials such as superalloys which uses a high power laser to focally heat a workpiece prior to material removal with a traditional cutting tool. A transient three-dimensional thermal model and cutting process model are developed using finite element analysis for LAML of Inconel 718.The paper aims to explore the thermal characteristics in LAML of Inconel 718 and the effects of laser power, laser moving speed and cutting speed on temperature and cutting force are investigated. In addition, a couple temperature fields of laser heating and cutting process which consider the heat generation associated with machining and laser heating is established and thus provide guidelines on parameter selection for future operations.
\end{abstract}

\section{Introduction}

Nickel-based superalloys with the performance of excellent hot hardness, thermal stability and thermal fatigue resistance are typical difficult-to-machine materials which are able to operate under extreme high temperature environment. Machining these materials results in high cutting forces, severe tool wear, high processing times and extremely cost-intensive. Laser assisted machining (LAM) which uses a high power laser to focally heat a workpiece prior to material removal with a traditional cutting tool. At elevated temperatures, the mechanical properties will change with yield strength decreasing thus reducing cutting force and tool wear, improving surface finish and increasing material removal rates. The feasibility and its advantages have been proved through the application of LAM .

By softening the material with a laser heat source, it was found the cutting forces were reduced by 20-40\% for turning of Inconel 718 [1] _ It was also observed that LAM improved surface finish and surface integrity for Inconel 718 [2]..Milling is a intermittent machining process, the impact of cutting tool and the workpiece makes the tool life less. The feasibility of LAML was first shown by König on satellite[3]. Shen [4] developed a finite element model to simulate the temperature and stress fields during LAML of silicon nitride. Tian [5] developed a transient, three-dimensional thermal model for LAML and successfully conducted experiments on silicon nitride ceramic and Inconel 718.

The key factor influencing the heating assisted machining is the control of temperature. However, the heating generation in cutting and laser heating is complex and hard to investigate by experimental research. In this study, heat transfer model and cutting process model are used to investigate the influence of operating parameters on removal temperature and heat transfer process.

\section{Transient Three-Dimensional FEA Model}

Fig. 1 illustrates the schematic of the laser assisted face milling of Inconel 718 in this study. 


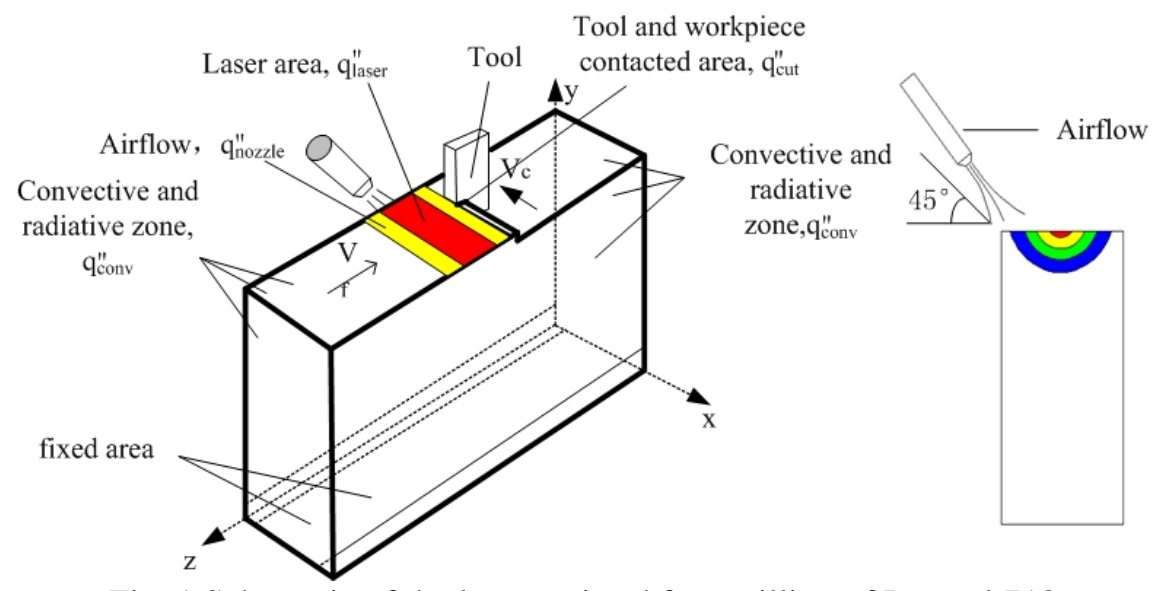

Fig. 1 Schematic of the laser assisted face milling of Inconel 718

In the process of laser assisted machining, maintaining material removal temperature within a proper rang is most important for achieving processing and obtaining good quality. The thermal model for the temperature distribution in the workpiece during LAML is based on a 3-D transient heat conduction analysis of a moving heat source applied to the workpiece surface.

Finite element method is used to discretize the governing equation. Temperature-dependent thermo-physical properties were used and updated at each step. The dimensions of workpiece is $50 \mathrm{~mm} \times 50 \mathrm{~mm} \times 6 \mathrm{~mm}$. For the symmetry with the plane that passes through the laser spot center, the half model is used and the mesh is refined within the range of laser spot and the depth of cut. The cutting speed is determined by the interval time of one load step. The initial workpiece temperature is set to $25^{\circ} \mathrm{C}$, the absorptivity of workpiece is 0.83 and the free convection coefficient is assumed to be $10 \mathrm{~W} / \mathrm{m}^{\circ} \cdot \mathrm{C}$.

The mesh model without cutting and the contour plot of the temperature distribution on workpiece after $20 \mathrm{~mm}$ laser spot moving is shown in Fig. 2 for the case of $250 \mathrm{~W}$ laser power, and $0.4 \mathrm{~mm} / \mathrm{s}$ laser moving speed. The surface heated by laser spot conducted energy to the interior of workpiece with laser moving and the temperature dropped with laser moving away. The temperature beneath the laser spot is very high and the gradient of temperatures around the laser spot is large due to the high intensity of the laser power and the low conductivity of Inconel 718. The temperature decreases with increasing distance from laser spot center.

An infrared thermometer was used to measure the temperature of the laser center which can be used as a standard to test the model and as a feedback to keep the temperature. The influences of different parameters on the measured temperature are shown in Fig.3.
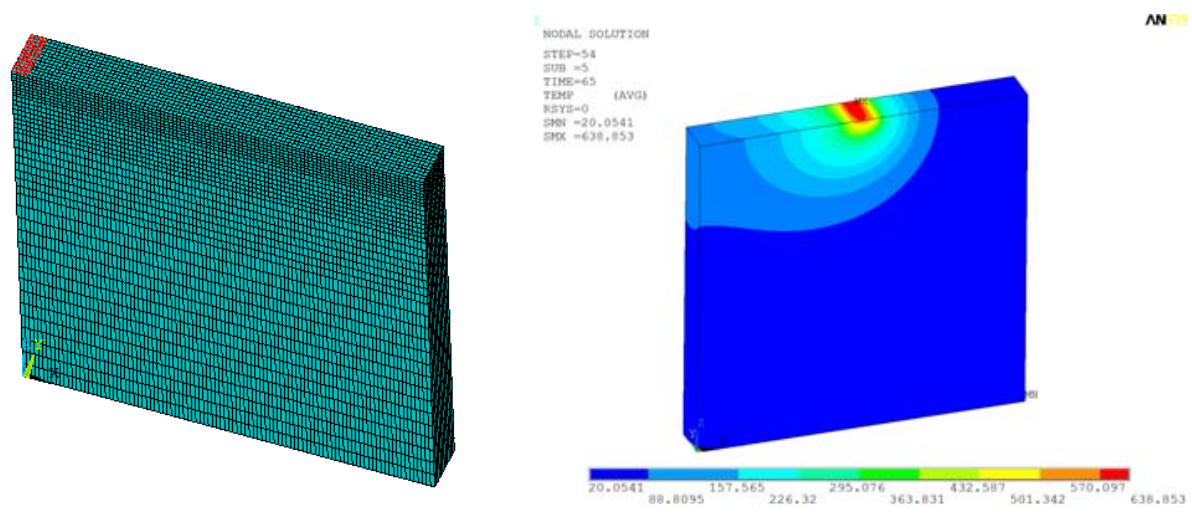

Fig. 2. The mesh model without cutting and the contour plot of the temperature distribution 


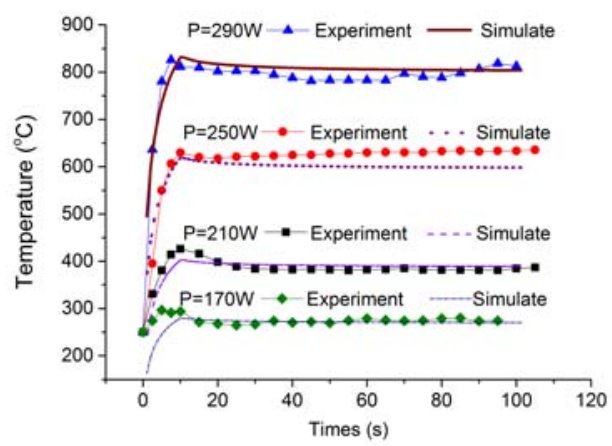

Fig. 3 The influences of laser power on the measured temperature

Among operating parameters, laser power has the most significant impact on the workpiece temperature, increasing laser power could increase the material removal temperature while causing high temperature gradient. Feed speed influenced the amount of energy deposited per unit time, the temperature increase with decreasing feed speed. However, the temperature changes little after a period of heating time when the workpiece change into the quasi-steady state. Generally, preheating cycle is an effective way to elevate the material removal temperature to the soften temperature before machining to reduce the amount of chipping on the tool for LAML. The experimental results are in good agreement with predictions calculated by the model, suggests that the model can predict the temperature distribution in laser assisted heating process very well.

\section{Model of Cutting Process}

During the LMAL process, materials impact by the cutting tool experience extremely high strain rate and the thermal induced from the laser heating is an effect which may reduce the flow stress at elevated temperature. In this study, JC model is used to investigate the stress and material since it is widely used for studying impact applications and is available in material model library in many commercial FEM codes such as 3D-wave advantage that was used in this study.

The simulation results are shown in Fig.4. The process of chip formation, cutting force and cutting temperature can be calculated by the model. The model is verified by the experiments, the main cutting force changes with rotating speed and laser heating temperature. The main cutting force increases with cutting speed increasing and decreases with laser heating temperature increasing as shown in Fig.4. The cutting zone temperature increase with laser power increasing improving the machinability of the material, the cutting force reduce for the soften of the material. The model also can be used to investigate the quantity of heat induced by cutting for different cutting speed and initial temperature heating by laser. The results is shown in Fig. 5.

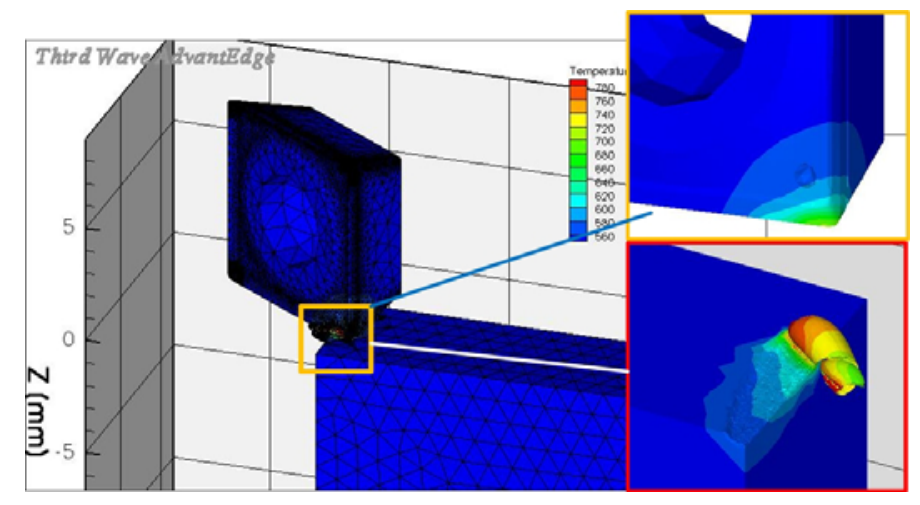

Fig. 4 Simulation results in the model of cutting process 

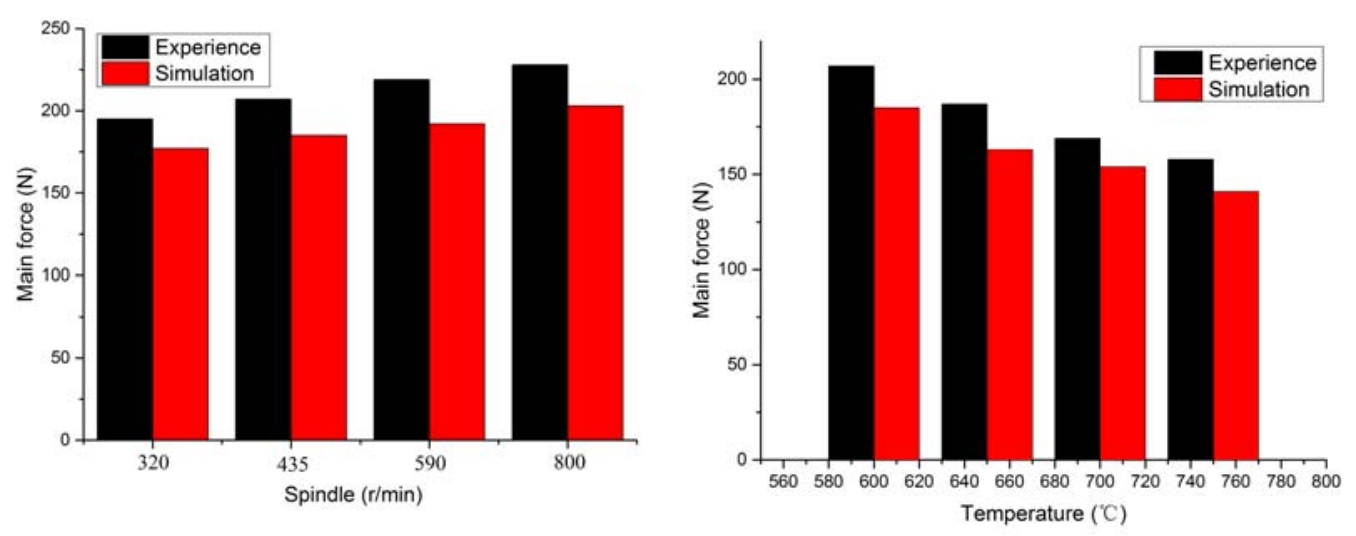

Fig. 5 Experience simulation results of the model of cutting

\section{Summary}

A transient three-dimensional heat transfer model for laser assisted milling of Inconel 718 is developed and validated through comparing the predicted temperatures with the temperature measured in experiments. The model can be used to evaluate the operating temperatures under various operating conditions. Laser power is one critical parameter for successful laser assisted milling operations. Laser power has the most great influence on the temperature of the workpiece and is the critical parameter for successful operation of LAML. Laser moving speed can affect the operating temperatures and has not big influence on temperature when the temperature is reaching steady state. The cutting force increase with cutting speed increasing and preheating temperature decreasing.

\section{Acknowledgment}

Financial support of this work by the National Science Foundation under Grant No. 51205097 and China Postdoctoral Science Foundation under Grant No. 2013M541401 are gratefully acknowledged.

\section{References}

[1] Rahman, M., Seah, W. K. H., \& Teo, T. The machinability of inconel 718. Journal of Materials Processing Technology, 63(1), (1997)199-204..

[2] Anderson, M., Patwa, R., \& Shin, Y. C. Laser-assisted machining of inconel 718 with an economic analysis. International Journal of Machine Tools \& Manufacture, (2006)46(14), 1879-1891.

[3]KONIG, W. , A.K. ZABOKLICKI, Laser-assisted hot machining of ceramics and composite materials[C]. Proceedings of the International Conference on Machining of Advanced Materials, Gaithersburg, MD, USA July 20- 22, (1993)455-463.

[4] Shen, X., \& Lei, S. Thermal modeling and experimental investigation for laser assisted milling of silicon nitride ceramics. Journal of Manufacturing Science \& Engineering, (2009)131(5), 611-623.

[5] Tian, Y., Wu, B., Anderson, M., \& Shin, Y. C. Laser-assisted milling of silicon nitride ceramics and inconel 718. Journal of Manufacturing Science \& Engineering, (2008)130(3), 361-374.. 